\title{
Débit réservé et autres précautions piscicoles actuellement imposées lors de l'installation de microcentrales hydroélectriques en Auvergne-Limousin
}

\author{
Reserve flow and other precautions \\ to preserve fish life \\ at present imposed for micro-hydroelectric power stations \\ in the Auvergne-Limousin region of France
}

R. Cuinat

Délégué Régional
J. J. Demars

Ingénieur adjoint

Conseil Supérieur de la Pêche

\section{Introduction}

La croissance de la demande en énergie et du prix du pétrole a suscité, depuis 1973 , un nombre accru de projets hydro-électriques dans le Massif-Central.

Une grande partie de ces projets portent sur des installations de faible puissance, appelées "microcentrales".

Restituant intégralement les eaux turbinées, sans pollution, sans fumée, sans échauffement sensible et sans odeur, ces installations sont considérées par une partie du public comme une "solution propre" aux problèmes d'énergie.

La majorité des pêcheures, au contraire, considère qu'elles provoquent, surtout dans les cours d'eau à salmonidés, des nuisances importantes pour une faible énergie produite. Les conflits sont d'autant plus fréquents, dans le Massif-Central, que le nombre de projets de microcentrales s'y multiplie : dans les sept départements de notre Délégation Régionale, nous avons eu connaissance de 62 projets dans la dernière période de 6 ans, au lieu de 10 et 15 dans les deux périodes précédentes (1968-1973 et 1962-1967) ${ }^{(1)}$.

Pour les microcentrales créées depuis 1974, certaines précautions ont été prises, en vue de limiter les nuisances sur l'environnement aquatique. Ces précautions devraient être encore intensifiées, et surtout affinées, en s'appuyant sur des études quantitatives qui commencent actuellement dans notre région.

Ce document a été rédigé en 1980. Depuis sont intervenus le décret $n^{\circ} 81.375$ du 15 avril 1981 et la Circulaire Interminis térielle de mêtme date, qui visent à assurer une meilleure protection de l'environnement aquatique et qui interdisent même toute nouvelle installation hydróélectrique sur certains cours d'eau (ou parties de cours d'eau).

(1) Puy-de-Dôme (30 projets), Haute-Loire (22), Haute-Vienne (19), Cantal (18), Corrèze (14), Creuse (7), Allier (5).

N.B. : Certains projets n'ont pas été réalisés.

\section{Définition}

On appelle couramment microcentrale hydro-électrique toute installation, de puissance brute installée inférieure à $500 \mathrm{~kW}$, dont la création est soumise à autorisation préfectorale ${ }^{(2)}$. Une telle usine, de faible puissance, est le plus souvent exploitée par un producteur autonome qui, presque toujours, vend son courant à Electricité de France.

Notons que les installations supérieures à $500 \mathrm{~kW}$ nécessitent, elles, une concession : c'est-à-dire une décision ministérielle après enquête et procédure souvent longues (2 à 5 ans) et compliquées. Les concessions sont le plus souvent demandées par EDF.

Quant aux conséquences sur la vie aquatique, il n'y a pas de différence de nature, mais seulement d'importance, entre ces deux types d'installation.

Dans notre région de moyenne montagne, une microcentrale est constituée par :

- un barrage de prise d'eau, d'une hauteur généralement modeste (le plus souvent de 1 à $4 \mathrm{~m}$ );

- une chambre de mise en charge comportant des dispositifs de dégrillage et de dessablage ;

- un dispositif d'écoulement dérivant l'eau vers l'usine : canal à ciel ouvert, ou conduite forcée, parfois successivement les deux;

- une ou plusieurs turbines;

- un très court canal de fuite, l'exploitation de la chute se faisant presque toujours très près du cours d'eau.

Une telle installation fonctionne le plus souvent au fil de l'eau; en effet le barrage est généralement de trop faible hauteur pour assurer le stockage d'un volume appréciable.

(2) Depuis juillet 1980, la limite de puissance brute est passée à $4500 \mathrm{kVA}$. 

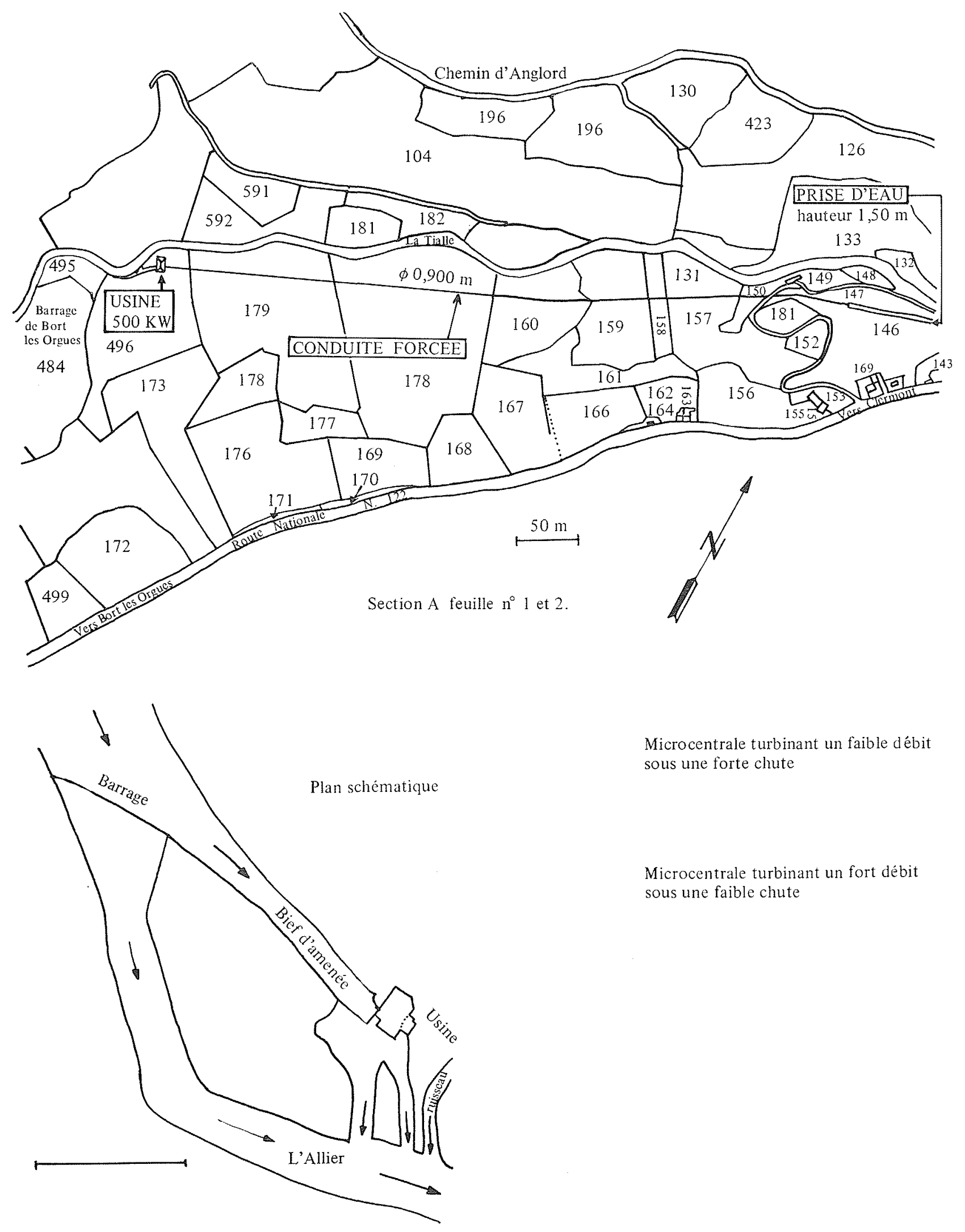

Microcentrale turbinant un faible débit sous une forte chute

Microcentrale turbinant un fort débit sous une faible chute 
La puissance brute d'une chute est égale au produit :

$$
P=Q \times H \times 9,81
$$

dans lequel :

$P=$ puissance brute, est exprimée en $\mathrm{kW}$;

$Q=$ débit dérivé maximal autorisé, en mètres-cubes par seconde ;

$H=$ hauteur de chute (dénivellation amont-aval), en mètres.

La puissance nette est égale à la puissance brute multipliée par un coefficient de rendement compris généralement entre 0,6 et 0,8 (pertes de charge dans les canaux ou conduites, rendement des turbines et des alternateurs).

$\mathrm{Si}$, théoriquement, peuvent exister toutes les situations intermédiaires entre une grande chute turbinant un petit débit et une faible chute utilisant un gros débit, la réalité montre que la plupart des installations se rattachent essentiellement à deux cas extrêmes :

- sur les cours d'eau importants, les usines, souvent anciennes, sont implantées à la place de vieux moulins (fig. 1); bénéficiant de débits relativement abondants, elles exploitent de petites chûtes obtenues par consolidation et rehaussement de la digue existante, l'eau étant amenée par un simple canal relativement court; - sur les petites rivières, les installations, très souvent récentes, ont été tout spécialement créées à des fins d'hydroélectricité (fig. 2); une longue conduite forcée métallique véhicule l'eau à turbiner; la puissance est alors généralement très proche du seuil des $500 \mathrm{~kW}$.

\section{Instruction des demandes d'autorisation}

Le pétitionnaire présente une demande au Préfet du département, en situant et décrivant son projet (tableau I). Cette demande est examinée par le Service chargé de la police de l'eau sur le cours d'eau concerné : généralement, la $\mathrm{DDA}^{(3)}$ pour les petits cours d'eau ou la DDE ${ }^{(3)}$ pour les grands. Pour cela, ce service consulte normalement divers organismes et usagers de l'eau, et notamment :

- le Service chargé de la pêche à la DDA;

- la Fédération Départementale des AAPP ${ }^{(4)}$.

- la Délégation Régionale du Conseil Supérieur de la Pêche.

Cette Délégation Régionale, après étude et enquête, formule au Service instructeur :

- son avis sur les conséquences piscicoles probables du projet;

- des propositions en vue de conserver au cours d'eau une vie aquatique et une valeur halieutique "raisonnables"; ces propositions concernent principalement le débit réservé et l'échelle à poissons;

- dans certains cas, des propositions motivées visant à refuser la demande, si les nuisances piscicoles parais-

(3) $\mathrm{DDA}=$ Direction Départementale de l'Agriculture ; DDE $=$ Direction Départementale de l'Equipement.

(4) AAPP = Association Agréée de Pêche et de Pisciculture. sent particulièrement importantes par rapport à l'intérêt énergétique du projet.

Après ces consultations, le Service instructeur élabore un avant-projet d'autorisation, assorti d'un "règlement d'eau'. Ce projet est mis à l'enquête (affichage en mairies concernées) et soumis à l'avis du Conseil Général. Ce n'est qu'à la fin de cette procédure qu'est donnée (ou parfois refusée) l'autorisation préfectorale.

Il est à noter en ce qui concerne les microcentrales que, depuis 1973, l'avis et les propositions formulés par la Délégation Régionale du Conseil Supérieur de la Pêche ont généralement été pris en compte par les services instructeurs des sept départements AuvergneLimousin.

Débit réservé, en vue du maintien d'une vie piscicole dans le parcours dérivé

\section{Nuisances provoquées par la réduction du débit}

Dans les installations anciennes, le règlement d'eau ne prévoit souvent qu'un débit réservé (ou "débit de franchise") très faible par rapport au débit naturel; parfois même aucun débit. La partie de cours d'eau entre le barrage de prise et la restitution des eaux turbinées est alors parcourue par un débit faible ou nul. sauf dans les périodes où le débit naturel est supérieur à la capacité de dérivation et de turbinage de l'usine. Il en résulte, dans le parcours dérivé, de nombreuses modifications, et particulièrement :

- une réduction des profondeurs et des vitesses de l'eau, ainsi que de la largeur mouillée; cette dernière est particulièrement diminuée dans les zones de "radiers" et il s'en suit :

a) une réduction de l'habitat disponible pour les poissons, principalement aux stades juvéniles qui ont besoin de zones peu profondes;

b) une réduction des zones convenant à la reproduction des salmonidés ou de la plupart des cyprinidés d'eau vive ;

c) une modification des peuplements d'invertébrés aquatiques, au détriment des espèces d'eau vive, d'où une diminution de leur biomasse totale et de la nourriture disponible pour les poissons;

d) qu'en outre, l'arrivée d'un débit de crue (très peu diminué par la dérivation), faisant brutalement suite à une période de très faible débit, peut arracher la faune benthique, non habituée à des vitesses importantes, et provoquer une stérilisation du milieu, jusqu'à ce que celui-ci se recolonise, ce qui nécessite plusieurs semaines;

e) que si la dérivation affecte une longue distance, les températures de l'eau peuvent être sensiblement modifiées; elles risquent notamment d'atteindre des valeurs excessives en été ;

f) qu'enfin, lorsque le turbinage est pratiqué "par éclusées" (fonctionnement intermittent, notamment lorsque le débit disponible est faible), le cours d'eau est également perturbé souvent très loin en aval du point de restitution, si les variations artificielles de débit ne sont pas amorties par une retenue de compensation. 
Tableau I. - Instruction d'une demande de microcentrale hydro-électrique : sehema.

Les nombres $(1,2 \ldots)$ indiquent l'ordre chronologique des consultations et de la préparation de l'au torisation.

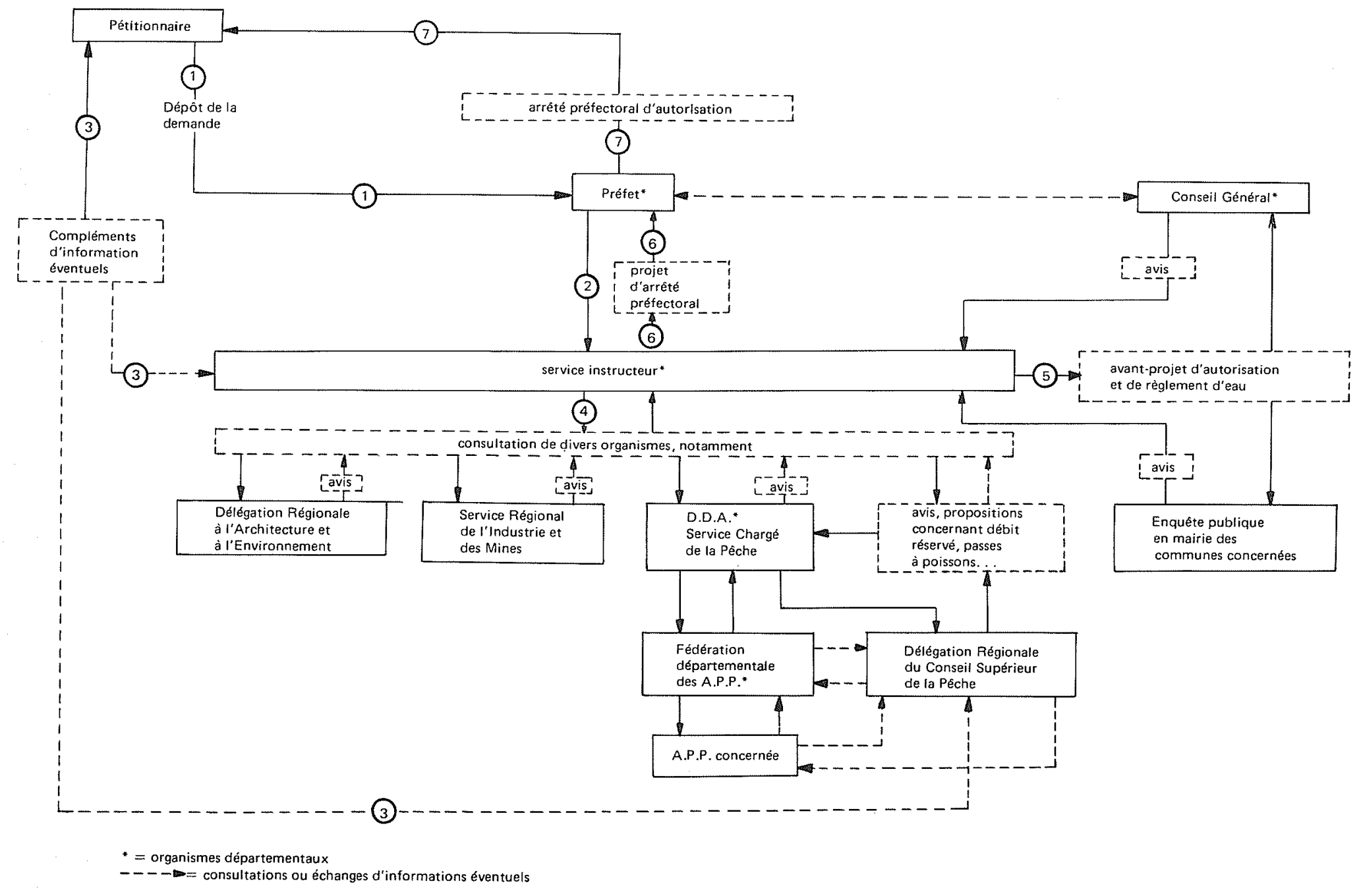




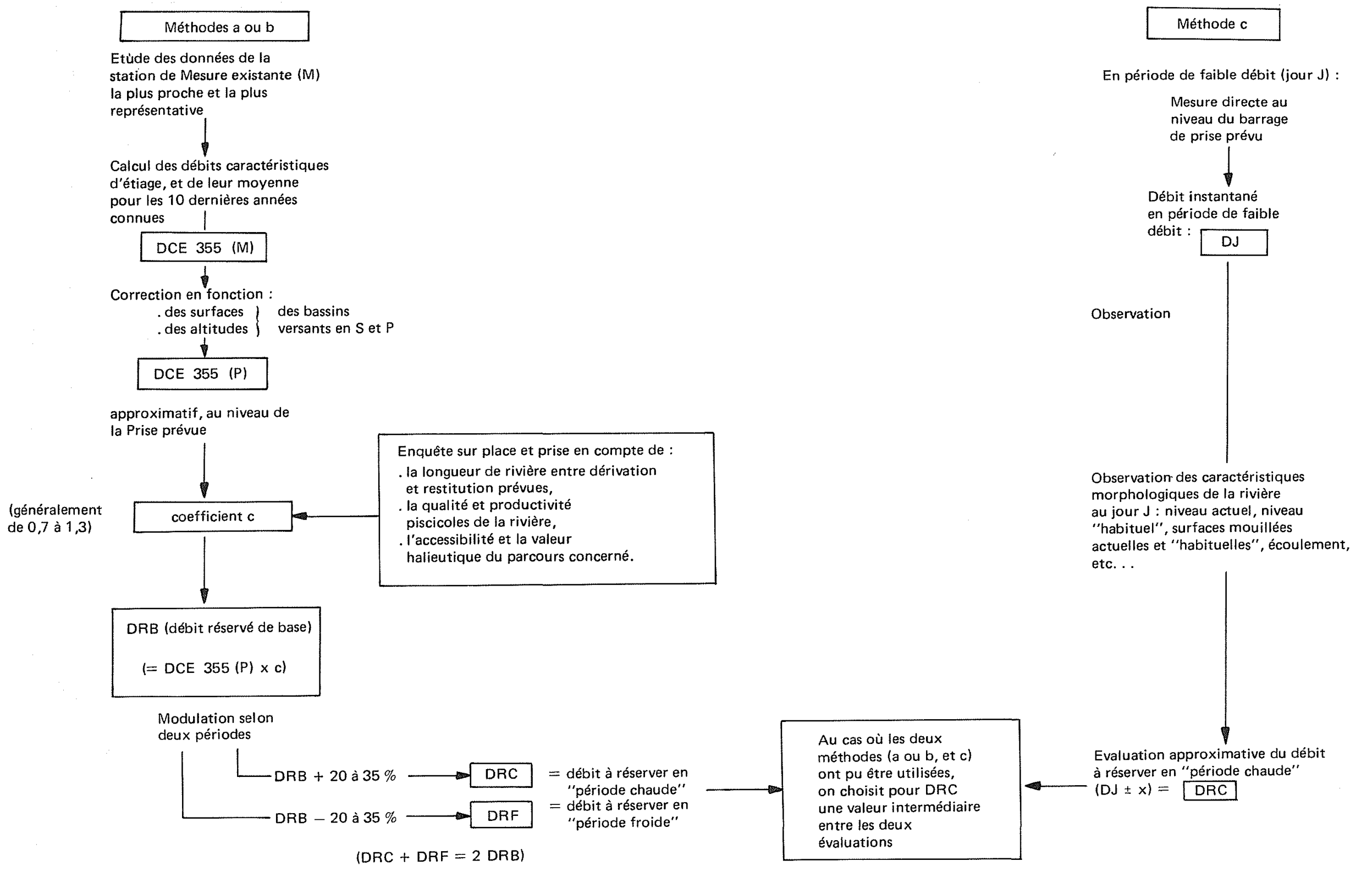


L'importance de ces diverses nuisances dépend beaucoup:

- de la taille du cours d'eau : après une même ponction relative de son débit, un ruisseau souffre généralement plus qu'une grosse rivière ;

- de la pente du cours d'eau et de la structure de son lit : un cours d'eau calme et profond, colonisé par une végétation aquatique abondante, sera beaucoup moins affecté qu'une rivière ou ruisseau à courant rapide, où dominent les radiers; c'est malheureusement ce deuxième type de cours d'eau qui fait l'objet de la plupart des projets de microcentrales.

Les exploitants de certaines de ces installations hydro-électriques versaient parfois aux APP concernées des "redevances compensatoires", permettant d'intensifier les repeuplements. L'expérience a montré que ces repeuplements ne résolvaient pas le problème, car la survie et la croissance des sujets introduits dépendent avant tout de la capacité d'accueil du milieu, elle-même conditionnée en grande partie par le débit réservé.

Les seuls repeuplements susceptibles, dans ce cas, d'améliorer la situation halieutique sont de type surdensitaire : c'est-à-dire un déversement de poissons de taille capturable destinés à être repris dans les jours qui suivent. Un tel procédé ne peut être considéré comme un remède à la dégradation du milieu aquatique.

\section{Fixation d'un débit réservé}

Pour tenter de préserver au milieu aquatique une qualité et une productivité "acceptables", dans le parcours dérivé, la $6^{\mathrm{e}}$ Délégation Régionale du Conseil Supérieur de la Pêche s'est efforcée, depuis 1973, de préconiser des valeurs de débits réservés adapté à chaque cas; ceci tout en restant entre des limites réalistes et "raisonnables", afin que ses propositions soient suivies, le plus souvent possible, par les services instructeurs et par les préfets. Il est évidemment rare que ces propositions satisfassent à la fois les pêcheurs (qui souhaiteraient préserver la quasi-intégralité du débit du cours d'eau) et des pétitionnaires (qui cherchent à rentabiliser au maximum leur projet, en dérivant et turbinant pendant la plus grande partie possible de l'année).

Pour cela, nous étudions successivement :

- le débit d'étiage du cours d'eau en question;

- la valeur moyenne de débit réservé souhaitable, par rapport à ce débit d'étiage et aux caractéristiques du cours d'eau et du projet ;

- la modulation possible de ce débit réservé moyen, selon deux périodes de l'année.

\section{A. Etude du débit d'étiage du cours d'eau}

Nous avons, jusqu'ici, pris en considération le DCE 355, c'est-à-dire le débit atteint ou dépassé 355 jours par an, ces jours étant consécutifs ou non. Nous calculons ensuite la moyenne de ces valeurs pour les dix dernières années connues.

a) Lorsqu'il existe une station de mesure sur le même cours d'eau, on calcule le DCE 355 moyen pour cette station; puis on corrige cette valeur en fonction des rapports des surfaces de bassins versants à la station et à l'emplacement prévu pour le barrage ; le cas échéant, on tient compte empiriquement des différences d'altitude (donc de pluviosité) entre les portions de bassins. Plus le site du projet est éloigné de la station de mesure, plus grands sont les risques d'erreur.

b) Lorsqu'il n'existe pas de station de mesure sur le cours d'eau, on se réfère à une ou plusieurs stations existant sur un cours d'eau le moins éloigné possible, et on corrige en fonction des surfaces de bassins versants. Les risques d'erreurs sont généralement encore plus importants que dans les conditions (a).

c) C'est surtout sur les "ruisseaux" que les évaluations de type (a) ou (b) sont souvent impossibles ou aléatoires. Nous préférons alors procéder à une mesure directe du débit, au niveau du projet de dérivation, dans une période sèche ${ }^{(5)}$. Dans ce cas, il est évidemment impossible de définir un DCE 355 mais on peut, par contre, à partir de la valeur mesurée, et en observant bien le cours d'eau, préconiser une valeur "raisonnable" pour le débit réservé.

Notons que la démarche (c) peut avantageusement s'ajouter à celles de types (a) ou (b).

\section{B. Evaluation du "débit réservé de base" (DRB) néces- saire (tableau II).}

Le débit réservé que nous proposons peut être inférieur ou supérieur au DCE 355 , selon :

- la longueur du parcours dérivé : lorsqu'il est très court (restitution au pied du barrage), le débit réservé peut être presque nul (valeur nécessaire au seul fonctionnement d'une passe à poissons); lorsqu'il dépasse trois kilomètres, comme c'est parfois le cas, il doit évidemment être beaucoup plus important ;

- la qualité piscicole de ce parcours, évaluée le mieux possible, à partir des données éventuellement disponibles, et compte-tenu du très court délai imparti; - sa valeur halieutique : accessibilité et proximité de grandes agglomérations sont également prises en compte.

En fonction de ces divers caractères la valeur du D.R.B. choisie est généralement comprise entre 0,7 et 1,3 fois le DCE 355 .

\section{Modulation selon les périodes de l'année}

Nos études de populations dans le Sud-Ouest et le Centre de la France ont montré que la croissance des truites était nulle ou très faible en automne et en hiver; elle s'effectue presqu'entièrement au printemps et en été, et plus particulièrement en avril, mai et juin (80 à $90 \%$ de la croissance totale annuelle). Il en est à peu près de même pour les invertébrés aquatiques, servant de nourriture à ces poissons. Il paraît donc prioritaire de demander une garantie de débits suffisants au printemps.

C'est en été, d'autre part, que la fréquentation touristique des cours d'eau est maximale; c'est aussi à cette période, où la température de l'eau est élevée,

(5) Ceci est malheureusement souvent impossible, dans le délai très court (un mois) qui nous est généralement laissé par le Service instructeur. 
qu'il faut éviter d'accentuer les baisses naturelles de débit.

Ainsi, nous préconisons le plus souvent un débit réservé modulé selon les deux périodes :

- période "froide" ( $1{ }^{\text {er }}$ octobre au 31 mars) : débit réservé $\simeq 66 \%$ du D.R.B. ;

- période "chaude" ( $1^{\text {er }}$ avril au 30 septembre) : débit réservé $\simeq 133 \%$ du D.R.B. (soit environ le double du débit réservé de période froide) ${ }^{(6)}$.

Il y a sur ce point conjonction d'intérêts avec les besoins en électricité, puisqu'EDF achète le courant "d'hiver" à un tarif presque double de celui "d'été".

\section{Critique de la méthode; discussion}

Cette démarche a été élaborée empiriquement, alors que nous ne disposions pas d'observations quantitatives des réductions de débit sur les effets piscicoles. La méthode devra être affinée dans les prochaines années, compte-tenu des connaissances acquises progressivement, sur des cours d'eau de types divers.

- Sur les petites rivières, où il n'existe généralement pas de stations de mesure, il semblerait préférable de renoncer à évaluer le DCE 355 (calcul trop aléatoire); il est beaucoup plus sûr de procéder à une (ou si possible plusieurs) mesure directe de débit en période estivale, assortie d'observations sur les niveaux, profondeur d'eau, vitesses du courant, etc., et d'en déduire directement les débits réservés nécessaires (méthode $c$, exemple 2).

- Sur les cours d'eau où des enregistrements sont disponibles, il serait bon de prendre en compte non seulement le DCE 355 mais aussi le module. Faute de quoi nous tendrons à défavoriser certaines rivières où les sècheresses estivales sont particulièrement prononcées.

Dans tous les cas, la fixation du débit réservé devra prendre en compte, en plus des facteurs énoncés plus haut, la valeur du débit maximal dérivable autorisé (qui est prévue dans le projet, et qui sera de toute façon précisée dans l'autorisation).

Selon que cette valeur est faible ou forte par rapport au module, le débit réel dans le parcours affecté sera très fréquernment ou très rarement (en temps de crue uniquement) supérieur au débit réservé.

A ce titre, il conviendra généralement d'augmenter très sensiblement la valeur préconisée pour les débits réservés, puisque la limite de puissance soumise à autorisation est passée de 500 à $4500 \mathrm{~kW}$ depuis juillet 1980 .

\section{E. Dispositifs d'écoulement}

Leur bonne conception et leur facilité d'entretien sont déterminants. Nous proposons généralement :

1) L'écoulement d'une partie du débit réservé par la passe à poissons. En effet, la plupart des échelles à poissons ne fonctionnent correctement que dans des

(6) Dans certains cas, les autorisations préfectorales interdisent toute dérivation en été et imposent un débit réservé faible tout le reste de l'année. La protection de la faune aquatique risque alors d'être très insuffisante, du fait qu'elle néglige la période printanière, essentielle pour la production piscicole. limites de débit assez étroites. Et les vitesses risqueraient d'être excessives si la totalité du débit réservé transitait par l'échelle, sauf à lui donner les dimensions très importantes.

2) L'écoulement du complément de débit réservé par un pertuis réglable. Celui-ci doit se situer en profondeur, pour être le moins possible affecté par des variations de niveau dans la retenue. Il doit déboucher à proximité immédiate du pied de l'échelle à poissons, de façon à la rendre plus attractive.

Ce dispositif, aussi bien que l'échelle, doit être protégé contre les obstructions, les épaves, et les manœuvres de la part du public ou des braconniers.

\section{F. Respect des débits réservés; dispositif de contrôle}

Pour une notable partie des microcentrales, les associations de pêcheurs se plaignent de ce que les débits réservés prescrits ne sont pas respectés, surtout en été. En fait, la vérification nécessite l'emploi de matériel et de techniques dont ces associations ne disposent pas.

Pour réduire les risques de contestation, nous avons été amenés, pour tous les projets examinés depuis 1978 , à proposer l'installation d'une section de contrôle, à situer à faible distance à l'aval du barrage de dérivation. Ce dispositif, constitué généralement par un petit seuil bétonné comportant une échancrure, ne vise pas à mesurer les débits en crue, mais seulement à permettre un contrôle facile du bon écoulement du débit réservé total (échelle + pertuis). Pour cela, deux repères permettent une vérification rapide de chacune des deux valeurs du débit réservé ("hiver" et "été"), par lecture de la hauteur d'eau en amont immédiat du seuil.

Il serait souhaitable qu'un tel dispositif soit également imposé aux installations antérieures à 1978. De plus, il s'avèrerait nécessaire d'imposer à toutes les installations un dispositif simple de vérification du débit effectivement dérivé.

Deux autres problèmes restent aussi à résoudre : a) les organismes chargés de la Police de l'eau (DDA ou DDE) ne disposent pas du personnel suffisant pour contrôler, sur le terrain, un nombre de microcentrales qui s'accroît chaque année ;

b) les pénalités encourues par les contrevenants sont dérisoires, et nullement dissuasives ${ }^{(7)}$

Cette insuffisance des moyens de contrôle et l'inadaptation de la règlementation à la situation actuelle expliquent en partie l'hostilité des pêcheurs contre les microcentrales.

\section{Maintien des possibilités de circulation des poissons}

Dans beaucoup de réalisations anciennes, les migrations des poissons sont impossibles, sauf éventuellement lors de fortes crues. Or, ces migrations sont souvent nécessaires au maintien d'un bon niveau de population.

(7) La loi de juillet 1980 a alourdi les amendes encourues. 
Les truites doivent pouvoir remonter certains cours d'eau pour trouver, dans les zones supérieures, les meilleures conditions de reproduction (ces déplacements ont surtout lieu en automne, et en période de débit assez important). Chez le Saumon, les migrations entre la mer et les zones de frayères sont indispensables à la survie de l'espèce.

Ainsi, pour la plupart des projets qui nous ont été soumis depuis 1973, nous demandons que le Pétitionnaire prenne toutes mesures voulues pour que ses installations permettent la circulation des poissons. Il s'agit là d'une "obligation de résultat": le Pétitionnaire conservant la responsabilité de l'ouvrage qu'il réalise, de son entretien, de son règlage et de son alimentation en eau; il devra le modifier s'il s'avère bloquer la migration des poissons.

Cette possibilité de franchissement implique deux précautions importantes :

\section{La limitation de la hauteur du barrage de dérivation}

Des hauteurs supérieures à 3 ou 4 mètres posent des problèmes difficiles, et sont à éviter dans toute la mesure du possible. Au-dessus de 4 mètres, l'échelle à poissons doit être étudiée avec un soin tout particulier, et nécessite de toutes façons des bassins de repos intermédiaires.

\section{L 'établissement d'une passe à poissons}

Sur ce point, étant donné "l'obligation de résultat" à laquelle doit être assujetti le Pétitionnaire, nous ne lui formulons que des "conseils techniques", dans la mesure où lui-même ou le Service Instructeur nous les demande.

Il est à remarquer que, dans certaines microcentrales construites depuis 1973, une échelle à poissons a bien été réalisée, mais son fonctionnement est plus ou moins đéfectueux. Ceci généralement parce que le Pétitionnaire :

- n'a pas fait appel à un conseiller technique spécialisé ; - ou n'a suivi qu'approximativement les conseils qui lui ont été donnés.

Sans entrer dans les détails techniques, rappelons seulement les principes essentiels conditionnant lc bon fonctionnement d'une passe à poissons :

- être attractive : pied de l'échelle situé le plus près possible du barrage, et du côté le plus amont lorsque ce barrage est oblique ; débit complémentaire "d'attrait" débouchant à proximité de l'entrée de l'échelle;

- être efficace, c'est à dire à courant suffisamment ralenti pour que la majorité des poissons puissent la remonter ;

- être protégée des épaves, et être placée et conçue de façon à pouvoir être correctement nettoyée et entretenue; cet entretien, qui incombe évidemment au Pétitionnaire, est en fait trop souvent négligé.

Les échelles à bassins successifs, pour être efficaces, sont d'une réalisation souvent coûteuse ; elles fonctionnent mal lorsque le niveau amont s'élève sensiblement. Pour ces raisons, nous conseillons plutôt maintenant les échelles à ralentisseurs plans (latéraux) ${ }^{(8)}$, avec une pente inférieure à 15 pour cent. Lorsque le barrage est de faible hauteur, ou que l'on peut sans inconvénient réaliser une dérivation dans une rive, les passes rustiques (fond irrégulier, garni d'enrochements ou autres irrégularités) peuvent donner toute satisfaction, sous réserve que leur pente soit faible $(<5 \%)$.

Notons que lorsqu'un ou plusieurs obstacles infranchissables existent déjà à proximité du barrage, nous ne demandons pas la création d'un échelle à poissons.

Malgré la présence d'une échelle correcte au niveau du barrage de prise, certaines installations hydroélectriques perturbent sérieursement les migrations, du fait que les poissons sont attirés dans le canal de fuite, par un débit turbiné supérieur à celui transitant dans la partie du lit naturel court-circuitée. Pour limiter cet inconvénient, particulièrement grave dans les cours d'eau à saumons, il faudrait, faute de pouvoir augmenter suffisamment le débit réservé :

- soit exiger la création d'une deuxième échelle, débouchant à l'aval immédiat des turbines;

- soit faire placer des grilles interdisant la remontée des poissons dans le canal de fuite ${ }^{(9)}$.

Il est à noter enfin que, même considéré comme "franchissable", un barrage l'est en fait rarement pour 100 pour cent de la population migrante; il occasionne de toutes façons un retard (plus ou moins prononcé selon les conditions climatiques et hydrologiques). Ainsi, la multiplication d'installations hydro-électriques, même bien équipées de passes, sur un même cours d'eau, peut conduire à des blocages très sérieux de la migration. Pour cette raison, la création de nouvelles installations hydro-électriques - centrales ou microcentrales - est absolument à proscrire sur les cours d'eau où l'on veut sauvegarder ou restaurer une population de saumons.

\section{Autres précautions}

Outre les débits réservés et l'échelle à poissons, nous proposons généralement au Service instructeur diverses autres précautions à savoir :

\section{Le canal (ou la conduite forcée) de dérivation ne doit prélever que le débit à turbiner}

Un canal d'amenée à ciel ouvert comporte presque toujours un ou plusieurs déversoirs, évacuant l'excès d'eau vers la rivière. Cet excès d'eau doit être ramené le plus près possible à l'aval du barrage de prise, afin de ne pas en priver inutilement une grande longueur du parcours dérivé.

(8) Ce type de passe a été décrit très précisément par le CEMAGREF, (M. Larinier).

(9) Les microcentrales sont toujours munies de grilles à l'amont immédiat de la prise d'eau, en vue d'arrêter les feuilles, branches, etc. Il semblerait illusoire, sauf cas particulier, de faire protéger les prises d'eau par des grilles plus fines, en vue d'empêcher l'entrée d'alevins. 


\section{Un dispositif de sécurité doit limiter les réductions brusques de débit à l'aval de la restitution}

Lorsque, pour une raison quelconque, la microcentrale cesse temporairement de turbiner, le débit de la rivière, à l'aval de la restitution, est réduit à la valeur du débit réservé acheminé par le parcours dérivé. Lorsque ce parcours est de grande longueur, cette réduction peut durer plus d'une heure, et se faire sentir sur la rivière sur une grande distance à l'aval, ce qui peut, surtout si elle se produit fréquemment, perturber non seulement la vie aquatique et la pêche, mais tous les autres usages de l'eau.

Un dispositif de sécurité est donc nécessaire au niveau de l'usine pour laisser automatiquement passer un certain débit dans le canal de fuite, si les turbines s'arrêtent, et ceci pendant un temps qui dépend de la longueur du parcours dérivé. Notons qu'un tel dispositif est de toute façon utile pour éviter les "coups de bélier" dans la conduite forcée.

\section{Cession des droits de pêche}

Le Pétitionnaire achète ou loue toujours des parcelles de terrain riveraines, sur lesquelles se situeront ses installations (barrage et annexes, conduite forcée, usine, canal de fuite). Dans les cours d'eau du Domaine Privè, il devient ainsi dètenteur du droit de pêche sur tout ou partie du parcours concerné par son projet.

Généralement, il laisse ce droit de pêche au public, de façon tacite comme c'est le cas le plus fréquent dans le Massif-Central. Il nous semble toutefois nécessaire que cette cession soit régularisée par un bail écrit, donnant la location de ce droit de pêche pour le franc symbolique, pour toute la durée d'exploitation de la microcentrale. En effet, il serait tout à fait illogique et inéquitable qu'à une dévalorisation piscicole et halieutique inéluctable du parcours, du fait de la réduction du débit, risque de s'ajouter une interdiction ou une privatisation de la pêche, au détriment de la collectivité.

Il est, de plus, indispensable que des pêches électriques d'inventaires puissent être réalisées, le cas échéant, dans des parcours affectés par certaines microcentrales, afin de préciser progressivement les connaissances dans ce domaine.

\section{Conclusion}

Les installations hydroélectriques réalisées depuis le début du siècle jusqu'aux environs de 1970, comme leurs règlements d'eau, n'avaient indéniablement pas pris suffisamment en compte la protection de l'environnement aquatique, notamment dans les cours d'eau à salmonidés. La raréfaction ou la disparition du Saumon en France - alors qu'elle a pu être évitée dans d'autres pays tout autant équipés pour l'hydro-électricité en est un signe probant. L'hostilité actuelle de la plupart des groupements de pêcheurs à tout nouvel équipement en est un autre, à ne pas négliger maintenant que la pêche est une nécessité sociale et touristique importante.

$\mathrm{La}$ prise de conscience de l'importance de notre environnement naturel, dans la dernière décennie, s'est traduite par quelque améliorations : les représentants de la pêche et les hydrobiologistes sont maintenant consultés pour la plupart des projets ; leurs avis et suggestions sont mieux pris en compte, surtout lors des projets d'autorisations instruites au niveau départemental. La plupart des microcentrales créées dans le Massif-Central, depuis 1974, sont assorties d'une passe à poissons. Toutes sont tenues à un débit réservé qui maintient à la rivière une certaine vie piscicole.

Pourtant, il reste encore bien des problèmes à régler, notamment :

- des études sont nécessaires, pour mieux connaître l'influence du débit sur la qualité et la productivité piscicole d'un cours d'eau, selon les caractéristiques qui lui sont propres (largeur, pente, nature du fond... ) Il serait ainsi possible de prévoir avec plus de précision, pour chaque projet, les effets des diverses valeurs de débit réservé, et de fournir ainsi aux "décideurs" les possibilités de concilier "au moins mal" des intérêts généralement opposés. De telles études sont en cours en quelques régions de France; elles sont très avancées aux Etats-Unis.

- les moyens de contrôle et la législation devraient être améliorés, afin que les débits réservés fixés soient effectivement respectés, et que les contestations soient moins fréquentes.

Ces améliorations sont d'autant plus nécessaires que les installations hydro-électriques se multiplient, principalement sur les cours d'eau à truites. Et le seuil de l'autorisation passant de 500 à $4500 \mathrm{~kW}$, le débit dérivé et/ou la longueur de rivière affectée par chaque installation nouvelle risquent de devenir beaucoup plus importants encore. Le patrimoine naturel et le potentiel halieutique et touristique des régions de montagne risquent alors d'être de plus en plus sérieusement diminués.

La plupart des anciens moulins, faisant partie de notre paysage, faisaient généralement bon ménage avec nos rivières et leurs pêcheurs. Il n'en est plus de même à mesure que les barrages sont surélevés, que les biefs à ciel ouvert sont remplacés par des conduites forcées, et que la rivière n'est correctement alimentée en eau que les jours de crue. Une évolution inconsidérée dans ce sens, où la production hydro-électrique ne laisserait plus de place aux autres usages de l'eau, serait certainement dangereuse. De sérieuses réflexions, d'ordre hydrobiologique, technique, et socio-économique, sont nécessaires pour résoudre le plus sagement possible ce difficile problème. 


\section{Abstract \\ Legal minimum flow and other fish protection regulations applying to construction of small hydro-electric power stations in Auvergne and Limousin}

In France, authorization is required from the Prefect's office (department administration level) before a hydro. electric power station of less than $500 \mathrm{~kW}^{*}$ gross power ("micro-power station") can be built.

The legislation on this was made many years ago (Law of 1919) and is relatively liberal. Since 1974 , as a result of an increasing number of projects carried out in the seven departments of Auvergne and Limousin, and awareness of environmental protection problems, authorization have been generally accompanied by more stringent requirements to be complied with by the construction.

Until more accurate technical and scientific data become available on the extent to which these dams affect fishbreeding, a "basic legal minimum flow" (BLMF) is prescribed

* Since july 1980 , this limit is $4500 \mathrm{~kW}$ which may range from 70 to $130 \%$ of the characteristic rate of flow of the river. This coefficient is proposed by the regional board of the Firshery Office in accordance with quality of aquatic life, accessibility for angling and length of river diverted (generally several hundred metres for every $4 \mathrm{~km}$ ).

The basic minimum flow selected is regulated as follows:

A lower value (minimum $0.7 \mathrm{BMLF}$ ) during the cold season

A higher value (maximum $1.3 \mathrm{BMLF}$ ) during the warm season (april to september), when invertebrate production and trout-growth are at a high level, and electricity requirements are usually lower.

Construction of a fish-ladder and a simple flow control device is almost always required.

The authorities usually seek advice from anglers' associations and the regional board of the Fishery Office (technical department). 